\title{
CLINICAL VARIABILITY IN TWO MACEDONIAN FAMILIES WITH ARTERIAL TORTUOSITY SYNDROME
}

\author{
Kocova $\mathrm{M}^{1, *}$, Kacarska R ${ }^{1}$, Kuzevska-Maneva $\mathrm{K}^{1}$, Prijic $\mathrm{S}^{2}$, \\ Lazareska $\mathrm{M}^{3}$, Dordoni $\mathrm{C}^{4}$, Ritelli $\mathrm{M}^{4}$, Colombi $\mathrm{M}^{4}$
}

*Corresponding Author: Professor Dr. Mirjana Kocova, University Pediatric Clinic, Str Majka Tereza 17, 1000 Skopje, Republic of Macedonia. Tel: +389-70-242-694. Fax: +389-2-311-1713. E-mail: mirjanakocova@ yahoo.com

\begin{abstract}
Arterial tortuosity syndrome (ATS) is a rare autosomal recessive disorder caused by mutations in the solute carrier family 2 member 10 (SLC2A10) gene encoding a glucose/ ascorbic acid transporter. The clinical features of ATS are mild-to-severe tortuosity of the large and medium arteries throughout the body, accompanied by dysmorphisms and joint laxity. Vascular changes in different parts of the body lead to stenosis and/or aneurysms requiring difficult surgical procedures. Here we present two new patients with ATS from two unrelated families. Patient 1 presented at 10 years of age with headache and typical physical appearance, delicate skeleton, large visible pulsation of the carotid arteries in the neck, and joint laxity. On computed tomography (CT) angiography she had severe tortuosity of the aortal branches and cerebral arteries, but no significant tortuosity of the pulmonary arteries. Two cousins of the girl carried the same homozygous c.254T $>$ C, p.(Leu85Pro) mutation in $S L C 2 A 10$, however, they additionally had a severe involvement of the pulmonary vessels. Patient 2 was a 9-year-old girl diagnosed with severe tortuosity and stenosis of the pulmonary arteries and progressive myocardiopathy. Her physical appearance was very similar to Patient 1, except that she also had growth retardation. After long-term follow-up by cardiologists, she underwent cardiac surgery abroad, with an unfavorable outcome. Homozygosity for the c.685C $>$ T, p. $\left(\operatorname{Arg} 229^{*}\right)$ mutation in the SLC2A10 gene was detected. Consanguinity was
\end{abstract}

\footnotetext{
${ }^{1}$ University Pediatric Clinic, Skopje, Republic of Macedonia

${ }^{2}$ Division of Cardiology, Institute of Mother and Child, Belgrade, Serbia

${ }^{3}$ Institute of Radiology, Skopje, Republic of Macedonia

${ }^{4}$ Department of Molecular and Translational Medicine, Division of Biology and Genetics, School of Medicine, University of Brescia, Brescia, Italy
}

disclosed within both families. Our findings confirm the intrafamilial phenotype variability of ATS. A novel finding is the severe tortuosity of cerebral arteries causing migraine that has not been described before in a child with ATS.

Keywords: Arterial tortuosity syndrome (ATS); Cerebral artery; Consanguinity; Phenotype variability; SLC2A10 gene mutation.

\section{INTRODUCTION}

Arterial tortuosity syndrome (ATS, MIM 208050) is a rare autosomal recessive connective tissue disorder chiefly characterized by elongation and tortuosity of the large and medium sized arteries and anomalies of the vascular elastic fibers $[1,2]$. Alterations of the vascular morphology and structure lead to a propensity for aneurysm formation and vascular dissections, focal and/or widespread stenosis of the aorta and/or pulmonary arteries, and vasomotor instability. The first few years of life seem to be the most critical for potentially life-threatening events, particularly acute respiratory insufficiency and other complications related to pulmonary artery stenosis (PAS) [3]. Arterial tortuosity syndrome shares features with other connective tissue disorders, i.e., Marfan syndrome, Loeys-Dietz syndromes, Cutis laxa and Ehlers-Danlos syndromes, namely soft/ velvety/hyperextensible skin, facial dysmorphisms (i.e., elongated face, hypertelorism, beaked nose, high palate and/or bifid uvula, and micro/retrognathia), abdominal hernias, joint hypermobility, congenital contractures, scoliosis and other skeletal anomalies. Genetic analysis can help in delineating these entities [3]. Arterial tortuosity syndrome is caused by mutations in the SLC2A10 gene encoding the glucose/dehydroascorbic acid (DAA) transporter 10 (GLUT10) $[2,4]$. The exact role of GLUT10 as a DAA transporter in the pathogenesis of ATS remains to be clarified, but some evidence suggests that the short- 

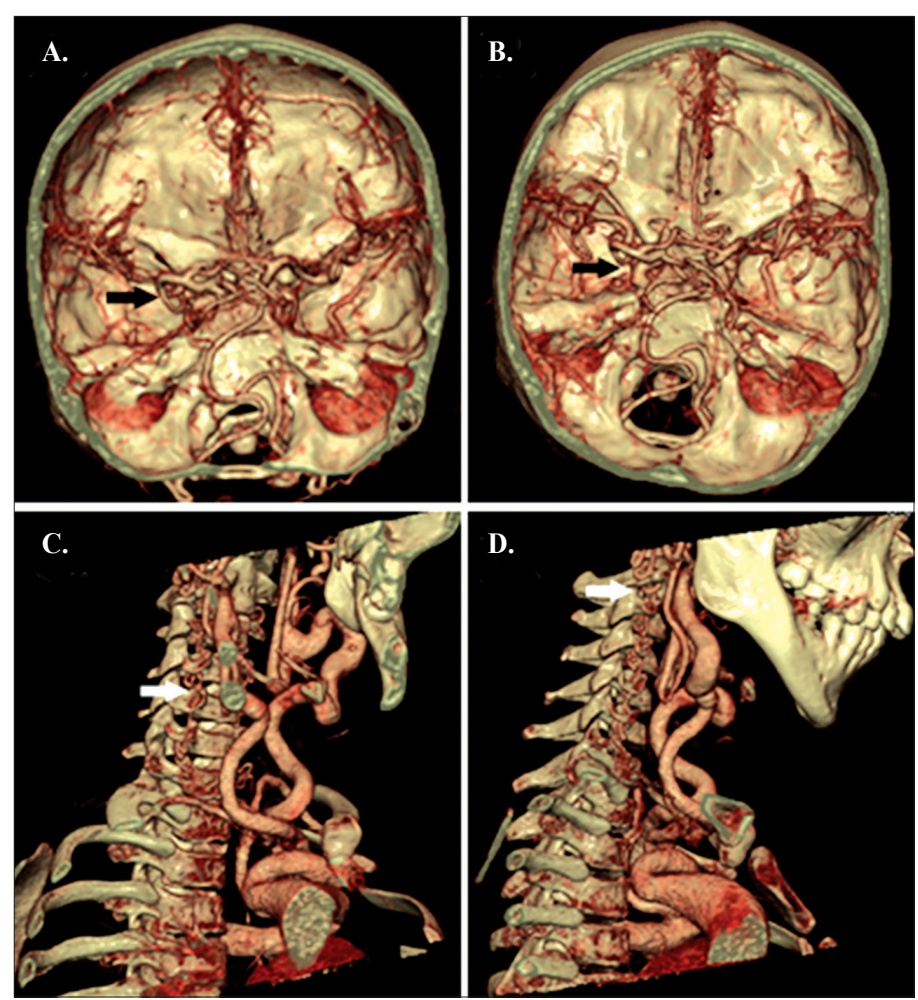

Figure 1. Color-coded volume rendering of the brain and neck CT-angiography of P1 with marked tortuosity of the cerebral arteries (black arrows) shown at different cross-sectional levels (A and B) and vertebral arteries (white arrows) shown in sagittal sections (C and D).
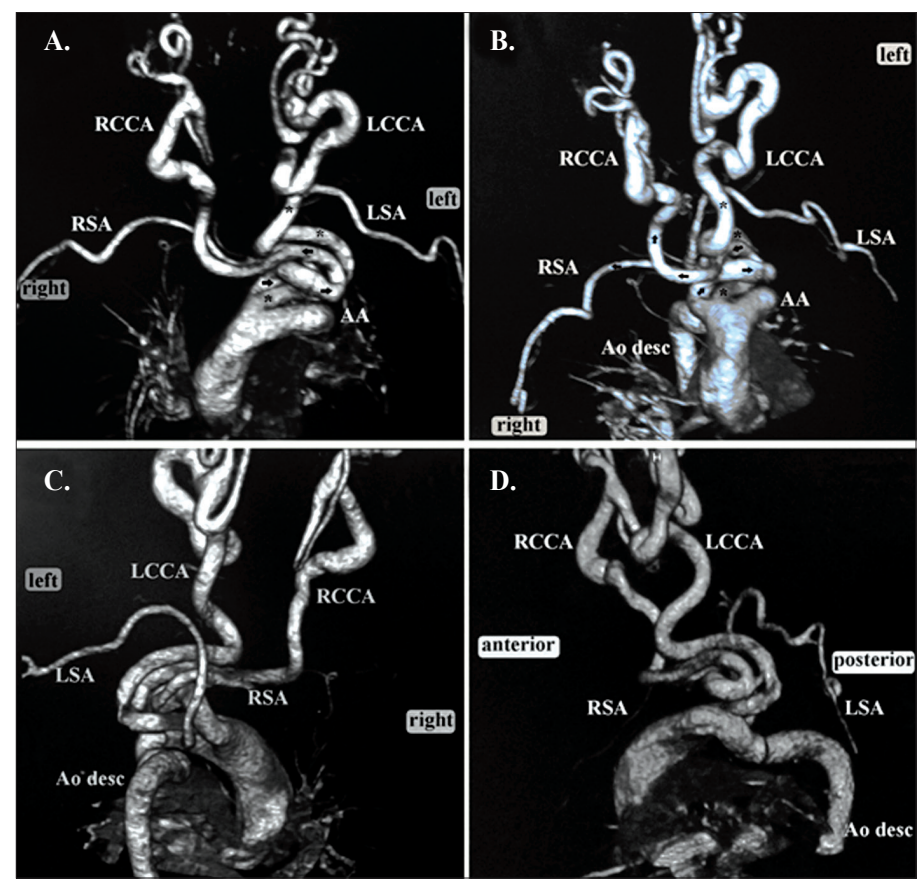

Figure 2. Computed tomography angiography of the aortic arch of P1 (A-D). Right subclavian artery (RSA) and right common carotid artery (RCCA) emerge from a right innominate artery, which is the first aortic arch branch (black arrows); left common carotid artery (LCCA) (marked with an asterisk) is the second and left subclavian artery (LSA) is the third aortic arch branch. age of ascorbic acid in the compartments of the secretory pathway can affect the production of extracellular matrix proteins both at epigenetic and posttranslational levels [4,5]. Here we present two unrelated patients with molecularly confirmed ATS, expanding the phenotypic spectrum and the variability of this rare disorder.

Mutation Screening. Genomic DNA was extracted from peripheral blood leukocytes using standard procedures; the exons and intronflanking regions of the SLC2A10 gene were amplified by polymerase chain reaction (PCR) and directly sequenced using an ABI PRISM ${ }^{\circledR} 3130 x \mathrm{x}$ genetic analyzer (Life Technologies, Carlsbad, CA, USA), as previously reported by Ritelli et al. [3]. The nucleotide and protein accession numbers correspond to the SLC2A10 (NM_030777.3; NP_110404.1) reference sequence. Mutations were annotated according to the Human Genome Variation Society (HGVS) nomenclature (www. hgvs. org/mutnomen).

\section{CASE REPORTS}

Patient 1 (P1). This 10-year-old Macedonian female was born to a healthy consanguineous couple following an uneventful pregnancy and delivery. Birth weight was $2600 \mathrm{~g}$ and birth length $48 \mathrm{~cm}$. A congenital diaphragmatic hernia was successfully treated at 15 days of age; subsequently, failure to thrive and poor suckling were noticed. At the age of 10 years, the patient was brought in for medical attention because of migraine, easy fatigability, and visible large carotid pulsations in the neck. Height and weight were within the normal range ( -1 and $-1.5 \mathrm{SD})$, respectively); a post-surgical atrophic scar on the abdominal wall was noticed as a residue of the surgical treatment in the neonatal period. Generalized joint hypermobility (Beighton score 6/9), high palate and dental crowding, flexible flat feet, and facial dysmorphisms, i.e., narrow, elongated face with beaked nose and long philtrum, were observed. Heart ultrasound was appropriate for sex and age (pulmonary pressure $20 \mathrm{~mm} \mathrm{Hg}$ ).

Magnetic resonance angiography (MRA), performed to investigate the migraine, brain and supra-aortic branches disclosed marked tortuosity of several vessels, including cerebral, carotid, and vertebral arteries (Figure 1). Computed tomography (CT) angiography revealed an aberrant ori- 
gin and tortuosity of the three branches of the aortic arch (Figure 2); no evident tortuosity of the pulmonary arteries was detected. Given the presence of widespread arterial tortuosity and peculiar facial dysmorphisms, ATS was suspected and confirmed by SLC2A10 molecular analysis that revealed the homozygous c. $254 \mathrm{~T}>\mathrm{C}$, p.(Leu85 Pro) missense mutation (Figure 3 ). This mutation was previously found in a homozygous state in two Macedonian brothers, 8 and 6 years old, with a full-blown clinical presentation of ATS including severe pulmonary arteries tortuosity (patients 2 and 3) [3]. Subsequently, a family pedigree reconstruction disclosed that the patients described by Ritelli et al. [3] and the present patient were second cousins (Figure 4).

Patient 2 (P2). This 9-year-old Macedonian female was born to consanguineous parents (second cousins) following an uneventful pregnancy and delivery. Weight and length at birth were appropriate for sex and age (3100 $\mathrm{g}$ and $50 \mathrm{~cm}$, respectively). Cyanosis after feeding was noticed in the first year of life and in-depth vascular studies with cardiac catheterization were performed that disclosed pulmonary hypertension $(65 \mathrm{~mm} \mathrm{Hg})$ due to PAS. Heart ultrasound showed right ventricular dilatation and hypertrophy with mild tricuspid regurgitation and mild mitral stenosis. Computed tomography angiography revealed dilatation of the pulmonary trunk $(29 \mathrm{~mm})$ and the left pulmonary artery $(32 \mathrm{~mm})$ and stenosis of the intra-parenchymal pulmonary arteries accompanied by severe tortuosity. The patient was followed by a cardiologist without any intervention until consultation at our hospital. On examination, the 9-year-old girl presented with short stature ( $-2 \mathrm{SD})$, low weight for sex and age ( $-3 \mathrm{SD})$, generalized joint hypermobility (Beighton score 8/9), facial dysmorphisms, e.g., elongated face, widely-spaced eyes,

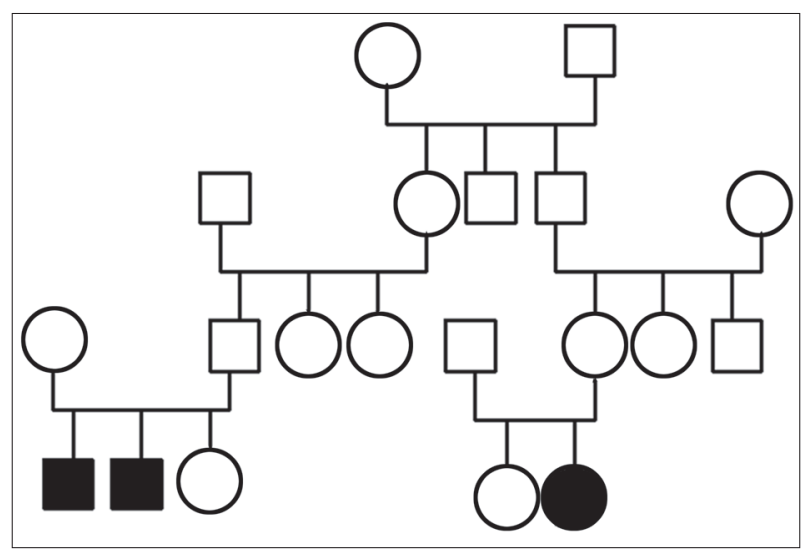

Figure 4. Family pedigree of P1. Children affected by ATS are shown in black, P1 is identified by an arrow.

beaked nose and micrognathia. She had systolic ejection murmur (4/6) in the precordium. This clinical presentation and previous cardiac findings were strongly suggestive of ATS. Molecular analysis of the SLC2A10 gene concluded the diagnostic process disclosing the previously described homozygous c.685C $>$ T, p.(Arg229*) nonsense mutation (Figure 3) [6,7]. Heart MRI confirmed severe right and moderate left pulmonary artery stenosis with severe dilatations and multiple stenoses of medium size arteries in the lung. The patient was sent to a cardiology hospital abroad for the second catheterization and surgical treatment. The catheterization was performed with the aim of balloon dilatation of the right pulmonary artery, however, due to the severe pulmonary arteries tortuosity and myocardiopathy with tricuspid regurgitation, she was scheduled for surgery. Tube graft of a decellularized patch was adapted to the right orifice of the pulmonary artery and side anastomosed to the common pulmonary artery. No intervention on the left pulmonary artery was performed. Intraoperative

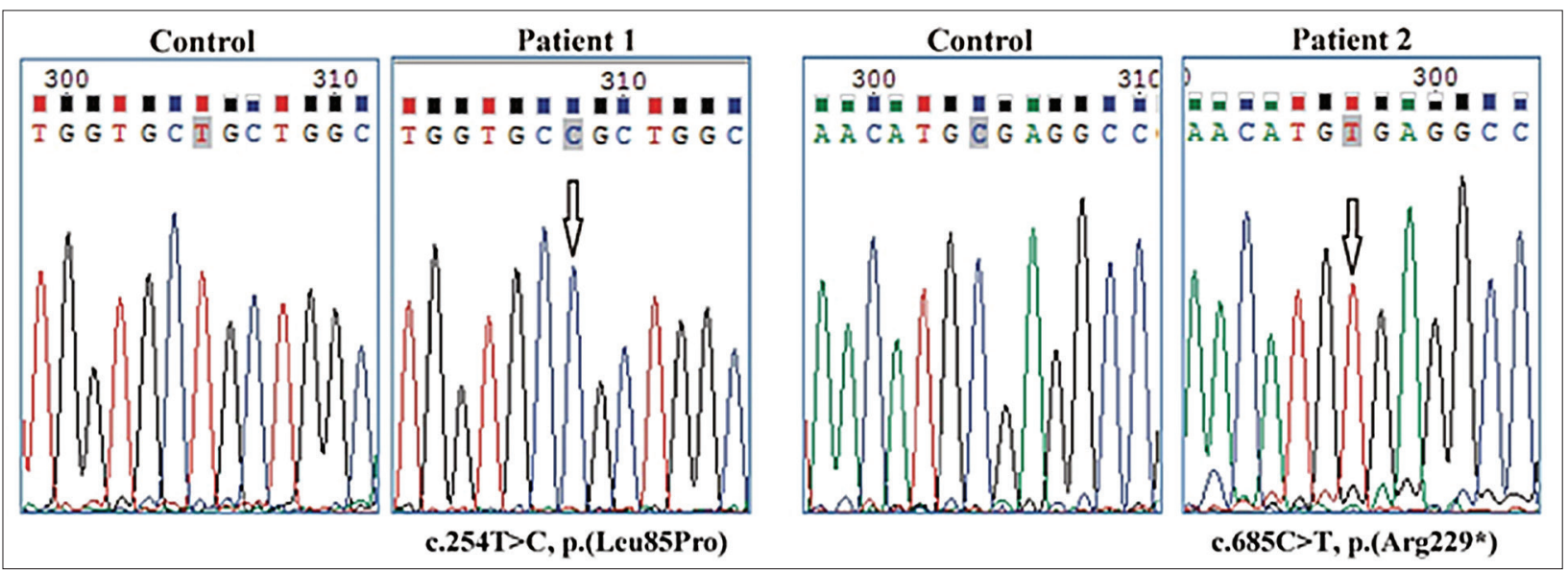

Figure 3. Molecular characterization. Sequencing of SLC2A10 exons and splice junctions revealed the following: P1 was homozygous for the $\mathrm{c} .254 \mathrm{~T}>\mathrm{C}$, p.(Leu85Pro) missense mutation and P2 was homozygous for the recurrent c.685C $>\mathrm{T}$, p.(Arg229*) nonsense mutation. 
examination also disclosed a major tricuspid failure and severe right ventricular dilatation; therefore, combined heart-lung transplantation was proposed. Unfortunately, the patient did not recover after surgery and died on the second postoperative day, after several resuscitation attempts, due to impaired contractile function of the heart, hemorrhagic event and cardiac arrest.

Informed Consent. Informed consent was obtained from all participants included in this study.

Ethical Approval. All procedures performed in studies involving human participants were in accordance with the ethical standards of the institutional and/or national research committee and with the 1964 Helsinki declaration and its later amendments or comparable ethical standards.

\section{DISCUSSION}

Arterial tortuosity syndrome is a rare and poorly characterized connective tissue disorder caused by recessive SLC2A10 gene mutations [1,2]. The estimated incidence ranges from 1:100,000 to 1:500,000 live births. To date, approximately 100 patients have been reported carrying some of the fewer than 30 described mutations [8]. Diagnostic criteria have not been standardized, however, widespread arterial tortuosity, PAS and specific facial dysmorphisms have been reported in the large majority of the patients, and are suitable to be considered as hallmarks of the disease [3]. The usual presenting symptom of ATS is PAS-induced cyanosis/respiratory failure in the first months of life. In the large majority of cases, the disease diagnosis is made in the first few years of life. In adulthood, life-threatening events appear to be rare; in elderly patients, chronic systemic and pulmonary hypertension, cardiac conductive defects, aortic root dilatation, stroke and intracranial aneurysms can be observed [1,3]. Some ATS patients require catheterization and/or surgery of severe PAS and/or progressive aortic root dilatation. Based on published data, in general, these procedures are well tolerated if performed in a timely manner, and surgery-related fatal events are rare.

Regarding prognosis, Wessels et al. [9] described a high infant mortality rate $(40.0 \%$ before the age of 5$)$ in ATS patients. Recent evidence suggests that life-expectancy in ATS is higher than initially reported $[3,9]$. The initial bias towards low life-expectancy/high mortality rate might be caused by the absence of molecular confirmation and the high rate of consanguinity in the studied families. Indeed, SLC2A10 molecular analysis is mandatory to conclude the diagnostic process and to distinguish ATS from other connective tissue disorders with common clinical signs and with high rates of life-threatening cardiovascular events, such as Loeys-Dietz syndromes or autosomal recessive Cutis laxa. Finally, in recent years, increased knowledge about the disease has facilitated targeted follow-up and timely cardiovascular surgery when needed, leading to an improved prognosis [3].

However, the clinical variability and range of severity in ATS patients is wide; the present cases exemplified a mild (P1) and a severe (P2) ATS clinical presentation. Patient 1 had an atypical presenting symptom, i.e., congenital diaphragmatic hernia. Diaphragmatic hernia has been reported in patients with connective tissue defects including ATS [10], but a definite suspicion/diagnosis of ATS in P1 was given only at 10 years of age, after in-depth vascular studies performed to investigate migraine. To the best of our knowledge, migraine has never been reported in ATS patients and the present data are not sufficient to conclude if this symptom is a result of the disease per se or has a different pathogenesis. Nevertheless, we can speculate that migraine might be part of the ATS spectrums, as cerebral hypo-perfusion and blood flow velocity changes are mechanisms involved in the pathogenesis of migraine and it is known that the tortuosity of cerebral arteries might lead to the above-mentioned brain blood flow changes [11,12]. The report by Pelaez et al. [13] of carotid artery, vertebral artery, and vertebro-basilar junction tortuosity on MRA imaging among patients with recurrent headache further supports the idea that tortuosity of the arteries responsible for brain perfusion may lead to migraine/headache. Furthermore, arterial tortuosity has been shown to be a risk factor for cerebral ischemic events and extracerebral dissection of arteries [14]. Moreover, several patients with ATS and stroke have been reported [1,15]. Pulmonary artery stenosis was not present in P1, justifying the absence of neonatal cyanosis and the delay of the diagnosis. The two Macedonian brothers described by Ritelli et al. [3], second cousins of $\mathrm{P} 1$, presented with a complete phenotype with cyanosis in the first months of life due to PAS, widespread arterial tortuosity, right ventricular dilatation or hypertrophy, facial dysmorphisms and joint hypermobility. The intra-familial variability in the presentation suggests interactions with other modifiers. Furthermore, the c.254T $>$ C, p.(Leu85Pro) mutation found in this family seems to be a recurrent Macedonian SLC2A10 mutation, as at least three unrelated families are carriers of this specific pathogenetic variant (Figure 3). Keeping in mind the recessive nature of ATS, the other parents of the three children in this family are putative relatives.

Patient 2 had a full-blown clinical presentation with cyanosis related to severe PAS detected in the first year of life. She had multiple cardiac consultations with progres- 
sion of myocardiopathy, however, the intervention was considered impossible until the age of 9 years when she had severe cardiac failure and was referred immediately for cardiac surgery in a specialized center. Major cardiac surgery involved stent insertions and patching to correct the numerous pulmonary arteries stenoses, however, she died postoperatively due to severe myocardiopathy and heart failure. Callewaert et al. [6] reported a 12-year-old Belgian boy (family A) without PAS, with widespread arterial tortuosity, dermal dysplasia, facial dysmorphisms, skeletal abnormalities and joint laxity and the same homozygous mutation of P2. Ritelli et al. [7] reported an 8-month-old male baby (Patient 1) with the same mutation, in compound heterozygosity with a second nonsense mutation, who presented with severe PAS, pulmonary hypertension, marked tortuosity of the aortic arch, bilateral congenital scrotal-inguinal hernia and facial dysmorphisms.

Severe ventricular hypertrophy was described in some of the largest studies [9] and the delay of the surgical procedure might have been the reason for myocardiopathy and lethal outcome in $\mathrm{P} 2$. Both of our patients received a delayed diagnosis due to the rarity of the syndrome and unavailability of molecular testing. It seems that whenever a connective tissue disorder is detected, or early cardiac features appear, the diagnostic process should include SLC2A10 molecular analysis, since this syndrome might be more common that previously considered.

In conclusion, our report suggests that migraine may be part of the ATS spectrum and that further studies are needed to investigate the cerebral perfusion in patients with cerebral arterial tortuosity. Our data emphasizes the clinical variability and different outcomes of ATS, ranging from uncomplicated arterial tortuosity to early death due to cardiovascular complications. Further studies are needed to better define the natural history and guidelines for management of these patients.

Declaration of Interest. The authors report no conflicts of interest. The authors alone are responsible for the content and writing of this article.

\section{REFERENCES}

1. Callewaert B, De Paepe A, Coucke P. Arterial Tortuosity Syndrome. In: Pagon RA, Adam MP, Ardinger HH, Wallace SE, Amemiya A, Bean LJH, et al., Eds. Gene Reviews ${ }^{\circledR}$ [Internet] University of Washington, Seattle, WA, USA; 1993-2018 (initial posting November 13 2014) (https://www.ncbi.nlm.nih.gov/ books/NBK253404.)
2. Coucke PJ, Willaert A, Wessels MW, Callewaert B, Zoppi N, De Backer J, et al. Mutations in the facilitative glucose transporter GLUT10 alter angiogenesis and cause arterial tortuosity syndrome. Nat Genet. 2006; 38(4): 452-457.

3. Ritelli M, Chiarelli N, Dordoni C, Reffo E, Venturini M, Quinzani S, et al. Arterial tortuosity syndrome: Homozygosity for two novel and one recurrent SLC2A10 missense mutations in three families with severe cardiopulmonary complications in infancy and a literature review. BMC Med Genet. 2014; 15: 122 (http://www.bio medcentral.com/1471-2350/15/122.)

4. Németh CE, Marcolongo P, Gamberucci A, Fulceri R, Benedetti A, Zoppi N, et al. Glucose transporter type 10-lacking in arterial tortuosity syndrome-facilitates de-hydroascorbic acid transport. FEBS Lett. 2016; 590(11): 1630-1640.

5. Zoppi N, Chiarelli N, Cinquina V, Ritelli M, Colombi M. GLUT10 deficiency leads to oxidative stress and non-canonical $\alpha v \beta 3$ integrin-mediated TGF $\beta$ signalling associated with extracellular matrix disarray in arterial tortuosity syndrome skin fibroblasts. Hum Mol Genet. 2015; 24(23): 6769-6787.

6. Callewaert BL, Willaert A, Kerstjens-Frederikse WS, De Backer J, Devriendt K, Albrecht B, et al. Arterial tortuosity syndrome: Clinical and molecular findings in 12 newly identified families. Hum Mutat. 2008; 29(1): 150-158.

7. Ritelli M, Drera B, Vicchio M, Puppini G, Biban P, Pilati $\mathrm{M}$, et al. Arterial tortuosity syndrome in two Italian paediatric patients. Orphanet J Rare Dis. 2009; 4: 20 .

8. Albuisson J, Moceri P, Flori E, Belli E, Gronier C, Jeunemaitre X. Clinical utility gene card for: Arterial tor-tuosity syndrome. Eur J Hum Genet. 2015; 23(10). doi: 10.1038/ejhg.2014.294.

9. Wessels MW, Catsman-Berrevoets CE, Mancini G, Breuning MH, Hoogeboom JJM, Stroink H, et al. Three new families with arterial tortuosity syndrome. Am J Med Genet. 2004; 131(2): 134-143.

10. Zaidi SHE, Meyer S, Peltekova I, Teebi AS, FaiyazUl-Haque M. Congenital diaphragmatic abnormalities in arterial tortuosity syndrome patients who carry mutations in the SLC2A10 gene. Clin Genet. 2009; 75(6): 588-589.

11. Law-Ye B, Fargeot G, Leclercq D. Arterial spin labeling hypoperfusion in migraine aura. Headache. 2017; 57(6): 935-936. 
12. Shayestagul NA, Christensen CE, Amin FM, Ashina $\mathrm{S}$, Ashina M. Measurement of blood flow velocity in the middle cerebral artery during spontaneous migraine attacks: A systematic review. Headache. 2017; 57(6): 852-861.

13. Pelaez JM, Levine RL, Hafeez F, Dulli DA. Tortuosity of carotid and vertebral arteries: a magnetic resonance angiographic study. J Neuroimaging. 1998; 8(4): 235-239.
14. Wei F, Diedrich KT, Fullerton HJ, DeVeber G, Wintermark M, Hodge J, et al. Arterial tortuosity: An imaging biomarker of childhood stroke pathogenesis? Stroke. 2016; 47(5):1265-1270.

15. Cartwright MS, Hickling WH, Roach ES. Ischemic stroke in an adolescent with arterial tortuosity syndrome. Neurology. 2006; 67(2):360-361. 